talenta

\title{
RESPONSE OUTCOMES OF CONCURRENT CHEMORADIOTHERAPY (CCRT) FOR STAGE III NASOPHARYNGEAL CARCINOMA
}

\author{
Feri Danil ${ }^{*}$ \\ ${ }^{\text {I}}$ Otorhinolaryngology Department, Gatot Soebroto Central Army Hospital, Jakarta, Indonesia
}

\begin{abstract}
Introduction: Nasopharyngeal carcinoma (NPC) is a rare malignancy with an incidence of $0.5-2$ per 100,000 in Europe and the United States [1]. NPC has high prevalence in certain regions of the world, especially in Southeast Asia with a poor prognosis. In Indonesia, the prevalence of NPC is 6.2/100 000, with 13000 yearly new cases, [2]. NPC is the forth most cancer found in Indonesia.

Case Report: This paper reported a case of 39 years old man with nasopharyngeal carcinoma and subsequently underwent concurrent chemoradiotherapy (CCRT).

Conclusion: The purpose of this paper is to explain experience at one of the largest single-centre of weekly cisplatin concurrently with radiotherapy with potential to be an optimal therapeutic regiment in the nonsurgical management of squamous cell carcinoma of head and neck (SCCHN) clinical stage III.
\end{abstract}

Article Info

Keywords:

Nasopharyngeal carcinoma, stage III, concurrent, chemo radiotherapy

\begin{tabular}{l}
\hline *Corresponding author: \\
Address: ORL-HNS Department, Oncology \\
Division, Gatot Soebroto Central Army Hospital
\end{tabular}

e-mail: fdaniltop@gmail.com

\section{CASE REPORT}

Nasopharyngeal carcinoma (NPC) has low incidence in Europe and the United States but relatively common in Southeast Asia, Southern China, the Arctic, and North Africa, especially in Southern China [3, 4]. The primary and only curative treatment of this malignancy radiotherapy. However, sequential and/or concurrent chemotherapy is also majorly applied for the treatment of NPC due to its sensitivity for chemotherapy. It had been known that concurrent chemoradiotherapy (CCRT) with/without adjuvant chemotherapy is recommended for NPC cases with locoregionally metastasis while radiotherapy alone is suggested for stage I NPC patients. Stage II NPC is preferably treated with CCRT $[5,6]$. Chen et al. in their phase III randomized trial showed that adding concurrent chemotherapy to two-dimensional radiotherapy (2D-RT) in NPC treatment was significantly improved overall survival.[7].

Squamous Cell Carcinoma of the Head and Neck (SCCHN) has 550,000 cases worldwide annually [8]. Traditionally surgery and radiotherapy (RT) either alone for early stage disease or in combination for NPC cases with locoregionally metastasis were thought to have curative potential $[9,10]$.

Moertel et al. classifying the second primary tumor in the order in which the tumor appeared diagnosed simultaneously or during the first 6 months since the primary tumor was diagnosed, and the time the tumor was diagnosed after that time period [11].

The differential diagnosis between metastasis and primary malignancy remains one of the most challenging tasks. Differentiating malignant tumors that spread from local tumor growth has a great influence on the patient's prognosis and can rationally guide developing therapeutic strategies [12].

\subsection{Radiotherapy}

Nasopharyngeal cancer is largely a radioresponse type so radiotherapy has a very large role in the treatment of KNF for all stages without distant metastases. Patients with distant metastases can be treated with radiotherapy with the aim of local control for the improvement of the quality life of patients. The management of radiotherapy given in the form of external radiation with conventional 2-dimensional (2D) techniques, 3-dimensional conformal techniques (3DCRT) and Intensity Modulated Radiotherapy (IMRT) techniques. IMRT is the highest form of radial conformal technique that gives high doses to tumor targets while providing low dose conformity to the surrounding healthy tissue by using multiple beam directions which can provide radiation conformity according to the shape of the target [13].

The selection of radiotherapy techniques is based on clinical indications and modalities owned by each health institution. The higher the technique used, the radiation side effects will be lower. The side effects of xerostomia were lower in the IMRT technique compared to conventional $2 \mathrm{D}$ techniques which was $39.3 \%$ vs $82.1 \%$. The radiotherapy dose given when using conventional 2D techniques is 66 Gy at an early stage and 70 Gy at a higher stage with a dose per fraction of 2 Gy, carried out 5 fractions every 1 week. For the IMRT technique the total dose given is 70 Gy in 33 fractions (2.12 Gy per fraction) [14].

\subsection{Chemotherapy}

Management of advanced locoregional NPC is to use a combination of radiotherapy and chemotherapy. Chemotherapy can be given before, during, or after radiation or is known as neoajuvan, concurrent, and adjuvant chemotherapy. The choice of chemotherapy is individualized according to the patient's characteristics. Standard chemotherapy for chemoradiation in advanced local conditions is concurrent cisplatin with radiotherapy. Meanwhile for chemoradiation followed by adjuvant chemotherapy can be used cisplatin + radiotherapy followed by cisplatin/5-FU or carboplatin/5 FU. For neoadjuvant chemotherapy the regimen used is docetaxel/cisplatin/5 FI, docetaxel/cisplatin. Cisplatin/5 FU, cisplatin/epirubicin/paclitaxel which are then followed by concordant coordination with the administration of cisplatin or carboplatin weekly [15].

A 39 years old man was admitted to ENT-HN inpatients in RSPAD. He had complain reccurent spontaneous bleeding from his left nostrils for about 4 months, noise in his ears, stuffy nose, and snoring. No other complaint from her head, eyes or neck. For about 2 months after, he complain for a mass in his right neck and mild headache. He has overweight and history of smoking, drink alcohol, and consume salted fish Clinical examination show enlargement lymphnodes about $1 \mathrm{~cm}$ in level II. Picture 1. CT scan examination were performed January 2018 and show a mass in nasopharyngeal. 

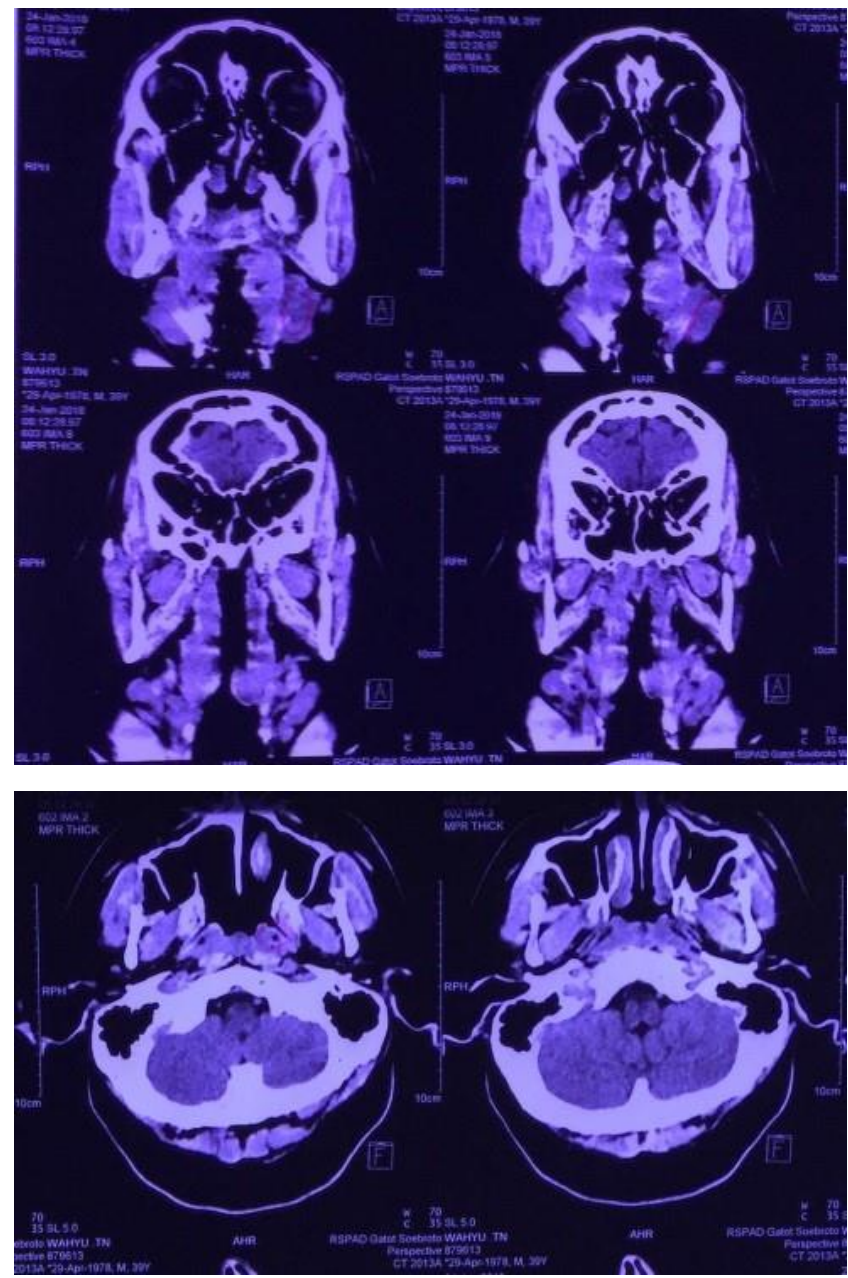

Picture 1. CT Scan nasopharyng before chemoradiation January 2018 (coronal and axial view)

Biopsy test was undifferentiated nonkeratinizing carcinoma of nasopharyngeal, (WHO-3) and pathologic uptakes in bilateral lymph nodes from level II to V. Staging were done and the patient was diagnosed with stage III nasopharyngeal carcinoma. Thereafter, the patient received one cycle concurrent chemoradiotherapy for around 3 months using Cisplatin $40 \mathrm{mg} / \mathrm{m} 2$ every week for 6 time and 7000 cGy radiotherapy with 200cGy for 35 shot per each. The chemoradioation finished in end June 2018 Radiological evaluation after concurrent chemoradiotherapy (CT Scan in October 2018) still indicates no progress as the result of the therapy but in clinical examination the patient show recovery progress. Picture 2 and 3 .

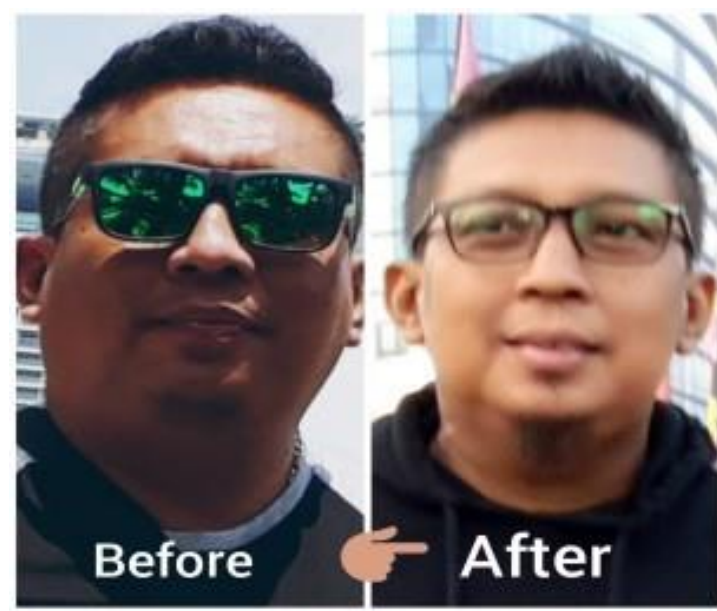

Picture 2. Patient profile before and after chemoradiotherapy.
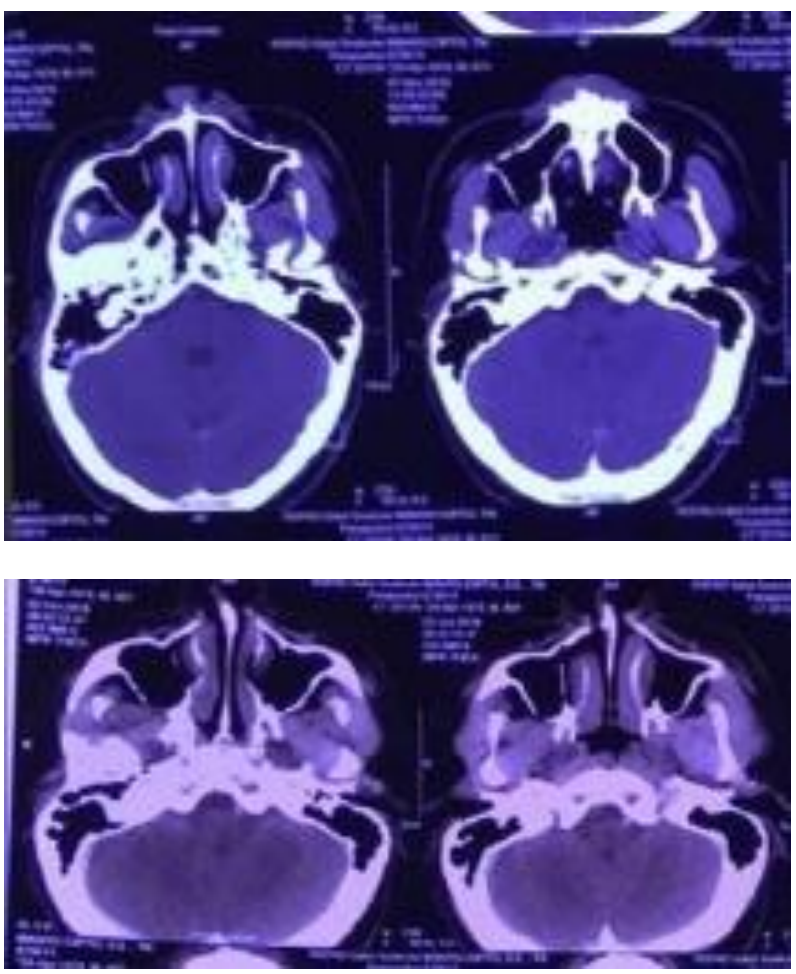

Picture 3. CT Scan nasopharyng after chemoradiation October 2018.

Thereafter, Whole Body PET CT-Scan was performed in October 2018 from laboratory Dharmais hospital to re-evaluate the radiological examination (CT-Scan from RSPAD, June 2018) and show complete response from the chemoradiotherapy that say no residive mass in nasopharyng, no bilateral juguler lymphadenopathy, no pathologic in head and neck area, lungs, mediastinum, abdomen, pelvic and nor the skeletal (Picture 4).

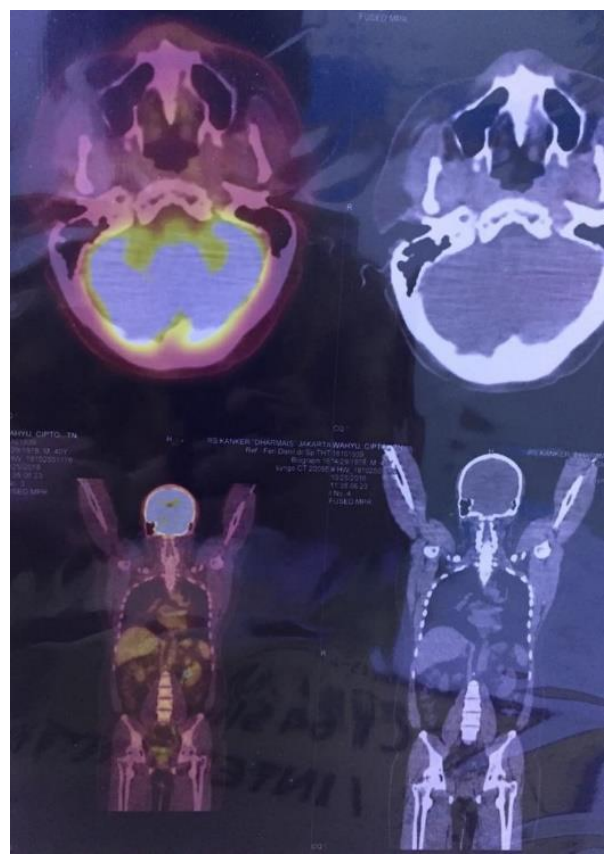

Picture 4. PET-CT Scan nasopharyng after chemoradiation October 2018 


\section{DISCUSSION}

The lateral wall of the nasopharynx is known to be the most common site of primary NPC, particularly the fossa of Rosenmüller. Symptoms and sign of NPC usually are nonspecific such as nasal obstruction, auditory abnormalities, headache, and cranial nerve palsies, mainly nerves III, V, VI, and XII.

The classification of NPC according to the World Health Organization (WHO) classification NPCs are classified into keratinizing squamous cell carcinoma (type I), differentiated non-keratinizing carcinoma (type II), and undifferentiated carcinoma (type III).

There, it is still controversy in the optimal medicine, time, dose and duration of chemotherapy. As is well known, three different ways to give chemotheraphy in addition with radiotherapy: before (neoadjuvant), while (concurrent), and after (adjuvant) radiation therapy.

The choice of treatment for locoregionally metastatic NPC cases are concurrent chemoradiotherapy (CCRT) with and without adjuvant chemotherapy. However, the stage I NPC cases is considered to be treated with radiotherapy alone and stage II NPC with CCRT. A phase III randomized trial by Chen et al. showed that NPC treatment by adding concurrent chemotherapy to two-dimensional radiotherapy (2D-RT) was significantly improved overall survival. For stage III/IV NPC, the chemotherapy combine with radiotherapy without metastasis based on consideration. The radiotherapy anti tumour strengthen simultaneously by radiosensitizer effect of chemotherapy. The systemic activity of chemotherapy would eradicate of the area outside chemotherapy micrometastasis. Finally, strengthen cell distribution which are sensitive to chemoradiotherapy and will increase transmission of the drug and result as the decrease the size of tumour size..

\section{CONCLUSION}

Concurrent chemoradiation is one of the treatments for undifferentiated nasopharyngeal carcinoma stage III that can improve survival of the patient.

\section{REFERENCE}

[1] Ferlay J. GLOBOCAN 2000. Cancer incidence, mortality and prevalence worldwide, version 1.0. IARC cancerbase. 2001.

[2] Adham M, Kurniawan AN, Muhtadi AI, Roezin A, Hermani B, Gondhowiardjo S, et al. Nasopharyngeal carcinoma in Indonesia: epidemiology, incidence, signs, and symptoms at presentation. Chinese journal of cancer. 2012;31(4):185.

[3] Chan A, Grégoire V, Lefebvre J-L, Licitra L, Hui EP, Leung S, et al. Nasopharyngeal cancer: EHNS-ESMO-ESTRO clinical practice guidelines for diagnosis, treatment andfollow-up. Annals of oncology. 2012;23:vii83-vii5.

[4] Network NCC. NCCN clinical practice guidelines in oncology (NCCN guidelines ${ }^{\circledR}$ ) head and neck cancers version 2.2017. 2017.

[5] Chen Q-Y, Wen Y-F, Guo L, Liu H, Huang P-Y, Mo H-Y, et al. Concurrent chemoradiotherapy vs radiotherapy alone in stage II nasopharyngeal carcinoma: phase III randomized trial. Journal of the National Cancer Institute. 2011;103(23):1761-70.

[6] Cheng SH, Tsai SY, Yen KL, Jian JJ-M, Chu N-M, Chan K-Y, et al. Concomitant radiotherapy and chemotherapy for early-stage nasopharyngeal carcinoma. Journal of Clinical Oncology. 2000;18(10):2040-5

[7] Xu T, Hu C, Wang X, Shen C. Role of chemoradiotherapy in intermediate prognosis nasopharyngeal carcinoma. Oral oncology. 2011;47(5):408-13.

[8] Ferlay J. Cancer incidence, mortality and prevalence worldwide. GLOBOCAN. 2004

[9] Forastiere A, Koch W, Trotti A, Sidransky D. Head and neck cancer. New England Journal of Medicine. 2001;345(26):1890-900.

[10] Chin D, Boyle GM, Porceddu S, Theile DR, Parsons PG, Coman WB. Head and neck cancer: past, present and future. Expert review of anticancer therapy. 2006;6(7):1111-8.

[11] Jones AS, Morar P, Phillips DE, Field JK, Husband D, Helliwell TR. Second primary tumors in patients with head and neck squamous cell carcinoma. Cancer. 1995;75(6):1343-53.

[12] Licciardello JT, Spitz MR, Hong WK. Multiple primary cancer in patients with cancer of the head and neck: second cancer of the head and neck, esophagus, and lung. International Journal of Radiation Oncology* Biology* Physics. 1989;17(3):467-76.
[13] Yoshizaki T, Ito M, Murono S, Wakisaka N, Kondo S, Endo K. Current understanding and management of nasopharyngeal carcinoma. Auris Nasus Larynx. 2012;39(2):137-44.

[14] Lo SS, Lu JJ. Natural history, presenting symptoms, and diagnosis of nasopharyngeal carcinoma. Nasopharyngeal Cancer: Springer; 2010. p. 41-51.

[15] Bailey BJ, Johnson JT, Newlands SD. Head \& neck surgery-otolaryngology: Lippincott Williams \& Wilkins; 2006. 